Article

\title{
Complementary Use of Presepsin with the Sepsis-3 Criteria Improved Identification of High-Risk Patients with Suspected Sepsis
}

\author{
Jong Eun Park ${ }^{1,2,+}{ }^{\mathbb{D}}$, Beomki Lee ${ }^{3,+}+\mathbb{D}$, Sun Joo Yoon ${ }^{4}$, Chi-Min Park ${ }^{5,6}$, Chul Won Jung ${ }^{7}$, Myung-Ju Ahn ${ }^{7}$, \\ Hyung-Doo Park ${ }^{3}$, Sung Yeon Hwang ${ }^{1}\left(\mathbb{D}\right.$, Tae Gun Shin ${ }^{1, *(\mathbb{C})}$ and Eun-Suk Kang ${ }^{3, * \mathbb{C}}$
}

1 Department of Emergency Medicine, Samsung Medical Center, Sungkyunkwan University School of Medicine, Seoul 06351, Korea; jongeun7.park@samsung.com (J.E.P.); sygood.hwang@samsung.com (S.Y.H.)

2 Department of Emergency Medicine, College of Medicine, Kangwon National University, Chuncheon 24289, Korea

3 Department of Laboratory Medicine and Genetics, Samsung Medical Center, Sungkyunkwan University School of Medicine, Seoul 06351, Korea; beomki.lee@skku.edu (B.L.); nayadoo@hanmail.net (H.-D.P.)

4 Department of Internal Medicine, Seoul National University Bundang Hospital, Seongnam 13620, Korea; yoonsunjoo@gmail.com

5 Department of Critical Care Medicine, Samsung Medical Center, Sungkyunkwan University School of Medicine, Seoul 06351, Korea; dr99.park@samsung.com

6 Department of Surgery, Samsung Medical Center, Sungkyunkwan University School of Medicine, Seoul 06351, Korea

7 Division of Hematology-Oncology, Department of Medicine, Samsung Medical Center, Sungkyunkwan University School of Medicine, Seoul 06351, Korea; leukemia1@skku.edu (C.W.J.);

check for updates

Citation: Park, J.E.; Lee, B.; Yoon, S.J.; Park, C.-M.; Jung, C.W.; Ahn, M.-J.; Park, H.-D.; Hwang, S.Y.; Shin, T.G.; Kang, E.-S. Complementary Use of Presepsin with the Sepsis-3 Criteria Improved Identification of High-Risk Patients with Suspected Sepsis. Biomedicines 2021, 9, 1076. https:// doi.org/10.3390/biomedicines 9091076

Academic Editor: Alessandro Russo

Received: 9 July 2021

Accepted: 21 August 2021

Published: 24 August 2021

Publisher's Note: MDPI stays neutral with regard to jurisdictional claims in published maps and institutional affiliations.

Copyright: (c) 2021 by the authors Licensee MDPI, Basel, Switzerland. This article is an open access article distributed under the terms and conditions of the Creative Commons Attribution (CC BY) license (https:// creativecommons.org/licenses/by/ $4.0 /)$ silk.ahn@samsung.com (M.-J.A.)

* Correspondence: taegunshin@skku.edu (T.G.S.); eskang@skku.edu (E.-S.K.); Tel.: +82-2-3410-2053 (T.G.S.); +82-2-3410-2703 (E.-S.K.)

$+\quad$ These authors contributed equally to this work.

\begin{abstract}
Presepsin has been proposed as an early indicator for diagnosis and prognosis in sepsis. We aimed to evaluate the prognostic accuracy of presepsin levels and additional value for identifying high-risk patients when taken together with the current sepsis criteria. This was a single-center, prospective, observational study of patients with suspected sepsis. The primary outcome was 28-day mortality. The prognostic performance of presepsin was evaluated by the area under the receiver operating characteristic curve (AUC), according to the sepsis definition using the Sequential Organ Failure Assessment (SOFA) score change (delta SOFA $\geq 2$ ) and lactate level $\geq 2 \mathrm{mmol} / \mathrm{L}$. A total of 755 patients were included. The AUC of presepsin for predicting 28-day mortality was 0.747 . Presepsin showed adequate prognostic accuracy regardless of the delta SOFA score or lactate level. High presepsin levels (>755 pg/mL) showed an independent association with 28-day mortality (adjusted odds ratio: 5.17), and significant differences in mortality were observed, even in patients with non-sepsis low lactate level. Compared with a single criterion of the delta SOFA score or lactate, the addition of the high presepsin criterion significantly increased discrimination. Presepsin showed fair prognostic performance regardless of the clinical sepsis criteria. Complementary use of presepsin with the Sepsis-3 criteria may identify more high-risk septic patients and provide useful prognostic information.
\end{abstract}

Keywords: presepsin; sepsis; septic shock; mortality

\section{Introduction}

The worldwide incidence of sepsis-related morbidity and mortality remains high [1]. To improve clinical outcomes, early identification of septic patients at high risk of mortality is critical. Nonetheless, an accurate and timely diagnosis of sepsis is challenging. In terms of pathophysiology, sepsis is defined as a life-threatening organ dysfunction caused by 
a dysregulated host response to infection [2]. A change in the Sequential Organ Failure Assessment (SOFA) score of two or more is the clinical surrogate criteria of sepsis and is associated with mortality of approximately $10 \%$ [2].

Along with clinical scoring, biomarker measurements can help physicians predict outcomes of patients with suspected infection and detect sepsis syndrome. Various circulating biomarkers have shown prognostic value in many studies, but few markers have been used in clinical practice due to the controversial issues in clinical value [3]. Among them, procalcitonin (PCT), lactate, and C-reactive protein (CRP) have been widely used for risk assessments of critically ill septic patients [4]. However, these biomarkers also have limitations, including the nonspecific nature of sepsis and the fact that no single biomarker is sufficient to represent all aspects of sepsis [5].

Presespin, also known as soluble CD14 subtype (sCD14-ST), is a fragment of CD14 that is released from activated monocytes following recognition of lipopolysaccharide (LPS) [6,7]. It has been suggested as an early indicator for diagnosis and prognosis in sepsis $[6,8]$. Compared to CRP and PCT, presepsin may be secreted earlier and can reach its peak faster $[6,8,9]$. Therefore, presepsin is expected to be a promising biomarker that could identify patients with sepsis who are at high risk for poor prognosis more quickly and sensitively. However, there have been conflicting results regarding the clinical value of presepsin $[4,10]$.

In this study, the primary aim is to evaluate the prognostic accuracy of presepsin in patients with suspected sepsis. We also investigated whether presepsin could be used complementarily with the current sepsis definition and lactate to identify high-risk patients.

\section{Materials and Methods}

\subsection{Study Design and Population}

This was a single-center, prospective, and observational study of patients with suspected sepsis who visited Samsung Medical Center-a 1989-bed, university-affiliated, tertiary care referral hospital located in Seoul, Korea. The study period was July 2020 through September 2020. We prospectively included consecutive patients according to the following inclusion criteria: age $\geq 18$ years old; suspected sepsis with procalcitonin measurement and blood culture test; and visited the emergency department (ED) or admitted to either the hematology-oncology department or the intensive care unit (ICU). We tested presepsin from 778 patients using residual plasma after procalcitonin measurement. Among the collected samples tested for presepsin, samples that fulfilled either of the following conditions were excluded: presepsin measured more than two $\mathrm{h}$ after measuring procalcitonin, or duplicate samples during a hospitalization period lasting less than one week. If there were multiple results for a patient, only the first test performed was incorporated in the analysis. The patients without blood cultures at the same time were also excluded. The Institutional Review Board of Samsung Medical Center approved this study (IRB No. SMC-2019-05-170). Informed consent was waived because this study was anonymous using residual samples.

\subsection{Measurement of Presepsin}

Samples were collected with a plasma separating tube (PST) containing sodium heparin anticoagulant (Vacutainer PST Tube $8.0 \mathrm{~mL}$, \#367964; Becton Dickinson [BD], Sunnyvale, CA, USA). Procalcitonin (PCT) was tested with Elecsys BRAHMS PCT assay (Roche, Basel, Switzerland) on Cobas e801 (Roche, Mannheim, Germany). After testing PCT, the residual samples were subjected to presepsin measurement with the PATHFAST ${ }^{\mathrm{TM}}$ assay kit (LSI Medience Corporation, Tokyo, Japan) and PATHFAST ${ }^{\mathrm{TM}}$ analyzer (LSI Medience Corporation, Tokyo, Japan) within two h. The PATHFAST ${ }^{\mathrm{TM}}$ presepsin assay is a cartridge-type, fully-automated enzyme immunoassay system that consists of magnetic particles coated with mouse monoclonal antibodies and alkaline phosphatase (ALP)-conjugated rabbit polyclonal antibodies [11]. The immunocomplex resulting from presepsin binding with the two antibodies generates luminescence after incubation with 
the chemiluminescent substrate [11]. The test requires $100 \mu \mathrm{L}$ as the minimum sample volume and takes about $17 \mathrm{~min}$ [11].

Prior to measuring the subjects' presepsin levels, laboratory performance validation of the PATHFAST ${ }^{\mathrm{TM}}$ assay kit and analyzer was conducted. Imprecision was assessed with two different levels of quality control (QC) materials measured in duplicate runs, twice a day, for five days. The results showed within-run coefficient of variation $(\mathrm{CV})$ and within-laboratory $\mathrm{CV}$ below $5 \%$. Linearity was evaluated with five different levels of verification control measured in four replicates. The coefficient of determination (R2) was 0.998 , and the $95 \%$ confidence interval (CI) for the slope of the linear regression model ranged from 0.904 to 1.073 .

Stability over time at room temperature was also evaluated in order to determine the exclusion criterion. A total of 45 samples evenly distributed along the measuring range (low, $<300 \mathrm{pg} / \mathrm{mL}$; medium, $\geq 300 \mathrm{pg} / \mathrm{mL}$ and $<1000 \mathrm{pg} / \mathrm{mL}$; and high, $\geq 1000 \mathrm{pg} / \mathrm{mL}$ ) were tested $0,1,2$, and $3 \mathrm{~h}$ after testing PCT. The mean value for each sample was calculated, and the difference between each test and the mean value was determined. While the difference was less than $20 \%$ of the mean value until $2 \mathrm{~h}$ after testing PCT, the difference between the two tests exceeded $20 \%$ of the mean value after $3 \mathrm{~h}$. In our institute, PCT is a stat test which should be reported within $60 \mathrm{~min}$ after sample collection; thus, it was determined that presepsin tests performed more than $3 \mathrm{~h}$ after sample collection should be excluded.

\subsection{Data Collection and Definitions}

Along with procalcitonin and presepsin, clinical data including demographic data, comorbidities, suspected infection focus, vital signs, laboratory tests, and blood cultures were obtained. The nearest values from the time of procalcitonin measurement were used, and the SOFA score was calculated with them. Missing values were not imputed. According to the Sepsis-3 definition, sepsis was defined as an acute change in total SOFA score (delta SOFA) $\geq 2$ points consequent to the infection [2]. The baseline SOFA score was assumed to be 0 if it was unknown. Septic shock was defined as sepsis with persisting hypotension requiring vasopressors to maintain mean arterial pressure (MAP) $\geq 65 \mathrm{mmHg}$ and a serum lactate level $>2 \mathrm{mmol} / \mathrm{L}$ despite adequate volume resuscitation. The primary outcome was 28-day mortality.

\subsection{Statistical Analysis}

Standard descriptive statistics were used for all variables including baseline demographics and outcomes. Categorical variables were presented as numbers with percentages and compared using a chi-square test. Continuous variables were presented as medians and interquartile ranges (IQRs) and compared using the Wilcoxon rank-sum test. The prognostic performance of presepsin for 28-day mortality was assessed as the area under the receiver operating curve (AUC), sensitivity, specificity, positive predictive value (PPV), and negative predictive value (NPV). We used a nonparametric approach for the comparison of two AUCs [12]. The Youden index was used to determine the optimal cut-off value for presepsin. According to the cut-off level, patients were categorized into the high presepsin group or the low presepsin group.

A multivariable logistic regression model was developed to assess variables related to 28-day mortality. The goodness-of-fit of the final logistic regression model was assessed using the Hosmer-Lemeshow test. The results were described as adjusted odds ratio (aOR) with a $95 \%$ confidence interval (CI). A $p$-value less than 0.05 was considered to indicate a statistically significant difference. STATA 15 (Version 15.0, STATA Corporation, College Station, TX, USA) was used for statistical analysis.

\section{Results}

\subsection{Baseline Characteristics}

A total of 755 patients with suspected sepsis were included (Figure 1). There was no confirmed case of coronavirus disease 2019 (COVID-19) in this study. By hospital location, 
the proportions of patients were $72.5 \%(n=547)$ in the ED, 20.3\% $(n=153)$ in the general ward, and $7.3 \%(n=55)$ in the ICU. The 28 -day mortality was $13.5 \%(n=102)$. The baseline characteristics of all patients and a comparison between survivors and non-survivors are shown in Table 1. The median age was 63.2 years (IQR 51.9-72.1), and 56.3\% $(n=425)$ were men. The most common focus of infection was respiratory infection $(24.5 \%)$, followed by urinary tract infection (15.5\%) and gastrointestinal infection (14.2\%). Characteristics such as older age, male sex, malignancy, respiratory infection focus, and number of patients with sepsis were more frequently observed among non-survivors than in survivors. The SOFA score, lactate, CRP, and procalcitonin at enrollment were significantly higher in non-survivors than survivors.

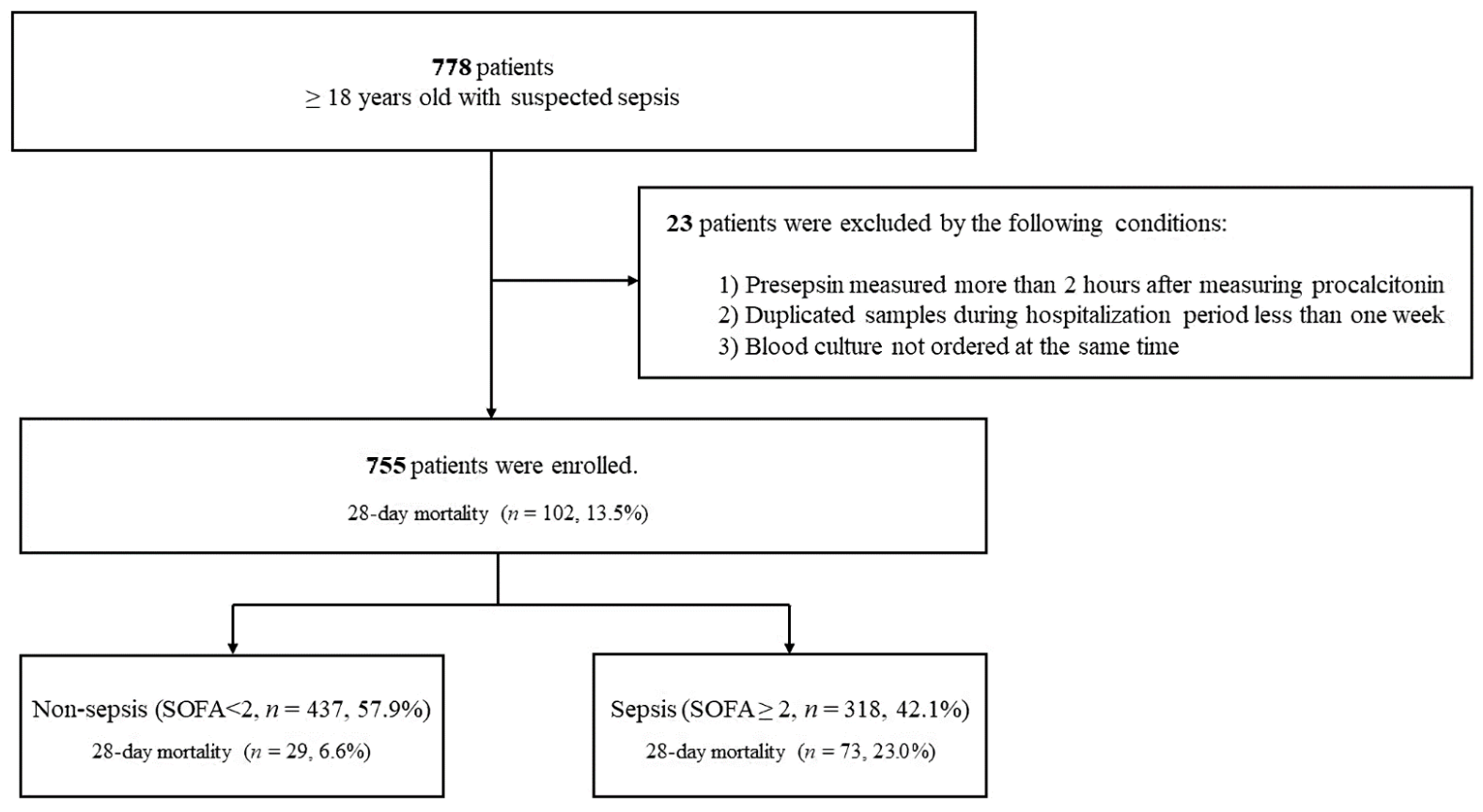

Figure 1. Study flowchart.

The number of non-sepsis, sepsis, and septic shock patients was 437 (57.9\%), 274 $(36.3 \%)$, and $44(5.8 \%)$, respectively, and the 28-day mortality in each of the three groups was $6.6 \%, 20.1 \%$, and $40.9 \%$, respectively. Baseline characteristics of the patients with non-sepsis, sepsis, and septic shock are shown in Supplementary Table S1.

\subsection{Presepsin Levels}

The median presepsin level was significantly higher in non-survivors than in survivors (1283 pg/mL (IQR, 765-3208) vs. 558 pg/mL [IQR, 315-1164], $p<0.001$ ) (Figure 2). The level of presepsin was highest in septic shock (1179 pg/mL (IQR, 643-3225)), followed by sepsis (933 pg/mL (IQR, 472-1861)) and non-sepsis (466 pg/mL (IQR, 284-938)), and there was a statistically significant difference in each group. Among sepsis patients, the presepsin level of septic shock patients was also significantly higher than in patients without shock $(p=0.003)$. The presepsin levels were still significantly different according to survival and the sepsis criteria, regardless of the presence of monocytopenia $\left(<500 \times 10^{3} / \mu \mathrm{L}\right)$. In patients with monocytopenia, the median presepsin levels were $570 \mathrm{pg} / \mathrm{mL}$ (IQR, 295-1189) in survivors vs. $1438.5 \mathrm{pg} / \mathrm{mL}$ (IQR, 889-4311) in non-survivors $(p<0.001)$, and $441.5 \mathrm{pg} / \mathrm{mL}$ (IQR, 266-937) in non-sepsis patients vs. $1054 \mathrm{pg} / \mathrm{mL}$ (IQR, 505-1906) in sepsis patients vs. 1246.5 (IQR 732-3181) in patients with septic shock $(p=0.028)$. Presepsin level was also significantly higher in patients with bacteremia than in those without $(1214 \mathrm{pg} / \mathrm{mL}$ (IQR, 687-2713) vs. $562 \mathrm{pg} / \mathrm{mL}$ (IQR, 315-1173), $p<0.001)$. The difference in presepsin levels between gram-positive and gram-negative bacteremia was not statistically significant. 
According to the Youden index, the optimal presepsin cut-off point for predicting 28-day mortality was $755 \mathrm{pg} / \mathrm{mL}$.

Table 1. Baseline characteristics.

\begin{tabular}{|c|c|c|c|c|}
\hline & $\begin{array}{l}\text { Overall } \\
(n=755)\end{array}$ & $\begin{array}{c}\text { Survivors } \\
(n=653,86.5 \%)\end{array}$ & $\begin{array}{l}\text { Non-Survivors } \\
(n=102,13.5 \%)\end{array}$ & $p$-Value \\
\hline Age, years & $63.2(51.9-72.1)$ & $62.3(49.8-72.1)$ & $65.8(59.8-71.7)$ & 0.004 \\
\hline Sex, male & $425(56.3)$ & $352(54.0)$ & $73(71.6)$ & 0.001 \\
\hline \multicolumn{5}{|l|}{ Comorbidities } \\
\hline Diabetes & $153(20.3)$ & $131(20.1)$ & $22(21.6)$ & 0.725 \\
\hline Cerebrovascular disease & $63(8.4)$ & $57(8.7)$ & $6(5.9)$ & 0.334 \\
\hline Chronic cardiac disease & $101(13.4)$ & $83(13.5)$ & $13(12.8)$ & 0.840 \\
\hline Chronic lung disease & $41(5.4)$ & $34(5.2)$ & $7(6.9)$ & 0.492 \\
\hline Chronic liver disease & $101(13.4)$ & $83(12.7)$ & $18(17.7)$ & 0.173 \\
\hline Chronic renal disease & $74(9.8)$ & $66(10.1)$ & $8(7.8)$ & 0.472 \\
\hline Malignancy & $497(65.8)$ & $409(62.7)$ & $88(86.3)$ & $<0.001$ \\
\hline \multicolumn{5}{|l|}{ Infection focus } \\
\hline Respiratory & $185(24.5)$ & $138(21.1)$ & $47(46.1)$ & $<0.001$ \\
\hline Urinary tract & $117(15.5)$ & $107(16.4)$ & $10(9.8)$ & 0.087 \\
\hline Gastrointestinal & $107(14.2)$ & $94(14.4)$ & $13(12.8)$ & 0.657 \\
\hline Hepatobiliary and pancreatic & $101(13.4)$ & $88(13.5)$ & $13(12.8)$ & 0.840 \\
\hline Bone and soft tissues & $66(8.7)$ & $62(9.5)$ & $4(3.9)$ & 0.063 \\
\hline Others & $82(10.9)$ & $74(11.3)$ & $8(7.8)$ & 0.292 \\
\hline Positive blood culture & $110(14.6)$ & $85(13.0)$ & $25(24.5)$ & 0.002 \\
\hline Gram-positive bacteria & $35(4.6)$ & $27(4.1)$ & $8(7.8)$ & 0.098 \\
\hline Gram-negative bacteria & $72(9.5)$ & $58(8.9)$ & $14(13.7)$ & 0.121 \\
\hline Fungus & $5(0.7)$ & $1(0.2)$ & $4(3.9)$ & $<0.001$ \\
\hline SOFA score & $2(1.0-5.0)$ & $2(1.0-4.0)$ & $5(3.0-9.0)$ & $<0.001$ \\
\hline Patients with sepsis & $318(42.1)$ & $245(37.5)$ & $73(71.6)$ & $<0.001$ \\
\hline Patients with septic shock & $44(5.8)$ & $26(4.0)$ & $18(17.7)$ & $<0.001$ \\
\hline \multicolumn{5}{|l|}{ Laboratory tests } \\
\hline $\mathrm{WBC}\left(10^{3} / \mu \mathrm{L}\right)$ & $7.8(3.9-12.4)$ & $7.7(4.0-12.0)$ & $9.3(3.1-17.4)$ & 0.030 \\
\hline Hemoglobin (g/dL) & $10.4(9.0-12.3)$ & $10.5(9.1-12.5)$ & $9.5(8.5-10.8)$ & $<0.001$ \\
\hline Platelet $\left(10^{3} / \mu \mathrm{L}\right)$ & $152(75.0-242.0)$ & $159(84.0-245.0)$ & $101(36.0-226.0)$ & 0.001 \\
\hline Total bilirubin (mg/dL) & $0.8(0.5-1.5)$ & $0.7(0.4-1.3)$ & $1.3(0.6-3.8)$ & $<0.001$ \\
\hline Creatinine (mg/dL) & $0.8(0.6-1.2)$ & $0.8(0.6-1.1)$ & $1.1(0.6-1.9)$ & 0.001 \\
\hline Lactate (mmol/L) & $1.5(1.1-2.3)$ & $1.4(1.1-2.1)$ & $2.4(1.7-4.4)$ & $<0.001$ \\
\hline $\mathrm{CRP}(\mathrm{mg} / \mathrm{dL})$ & $6.8(2.9-14.4)$ & $6.4(2.6-13.2)$ & $11.2(6.1-18.9)$ & $<0.001$ \\
\hline Procalcitonin (ng/mL) & $0.4(0.1-1.6)$ & $0.3(0.1-1.5)$ & $1.0(0.4-4.3)$ & $<0.001$ \\
\hline Monocytopenia & $348(46.1)$ & $298(45.6)$ & $50(49.0)$ & 0.524 \\
\hline
\end{tabular}

The data are presented as median (IQRs) for continuous variables or as numbers (\%) for categorical variables. IQR, interquartile range; SOFA, Sequential Organ Failure Assessment; WBC, white blood cell count; CRP, C-reactive protein.

\subsection{Multivariable Logistic Regression Analysis for 28-Day Mortality}

The results from univariable and multivariable logistic regression analysis for 28-day mortality are presented in Table 2. In multivariable logistic regression analysis, we adjusted predefined variables including age, sex, infection focus, SOFA score, lactate level, procalcitonin level, and comorbidities with a significant difference between survivors and non-survivors. A high presepsin level, over $755 \mathrm{pg} / \mathrm{mL}$, was significantly associated with 28-day mortality in both the univariable analysis (Odds ratio; OR 5.61, 95\% CI 3.44-9.16, $p<0.001$ ) and the multivariable model that adjusted for confounding factors (aOR 5.17, $95 \%$ CI 2.92-9.16, $p<0.001)$. The presence of malignancy, respiratory infection, delta SOFA score $\geq 2$, and lactate $\geq 2 \mathrm{mmol} / \mathrm{L}$ were also significantly associated with 28-day mortality. 
A

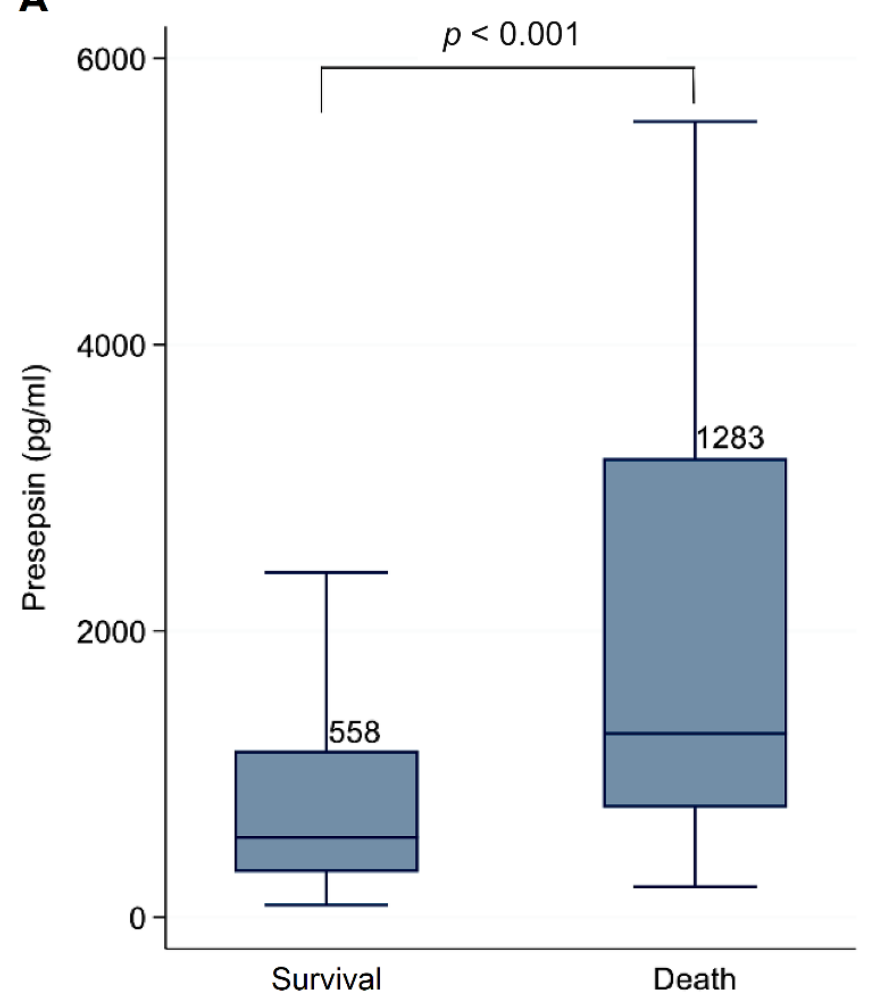

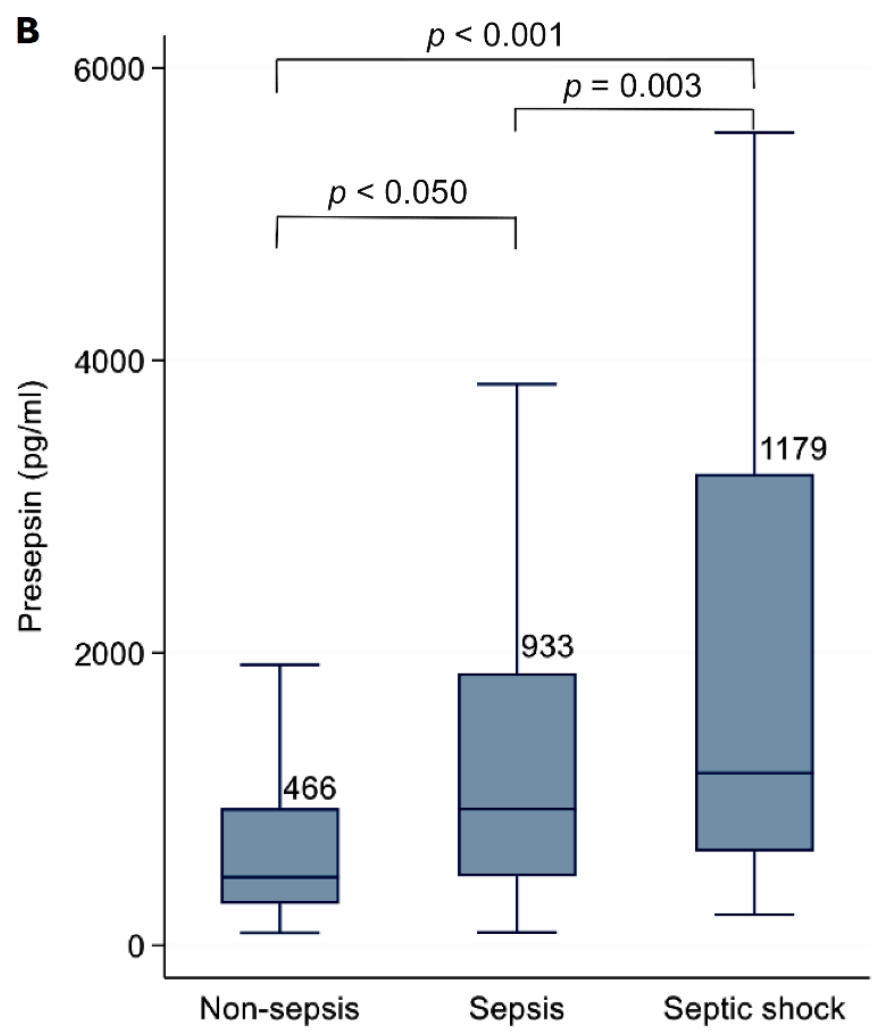

Figure 2. Comparison of presepsin levels according to (A) survival and (B) the sepsis criteria.

Table 2. Univariate and multivariable logistic regression analysis for 28-day mortality.

\begin{tabular}{ccccccc}
\hline & \multicolumn{3}{c}{ Univariable } & \multicolumn{3}{c}{ Multivariable } \\
\cline { 2 - 7 } & OR & $\mathbf{9 5 \% ~ C I}$ & $p$-Value & OR & 95\% CI & $p$-Value \\
\hline Presepsin $>$ 755 pg/mL & 5.61 & $3.44-9.16$ & $<0.001$ & 5.17 & $2.92-9.16$ & $<0.001$ \\
Age, years & 1.03 & $1.01-1.04$ & $<0.001$ & 1.02 & $1.01-1.04$ & 0.016 \\
Sex, male & 2.15 & $1.36-3.39$ & $<0.001$ & 1.14 & $0.67-1.95$ & 0.626 \\
Malignancy * & 3.75 & $2.09-6.74$ & $<0.001$ & 5.34 & $2.71-10.53$ & $<0.001$ \\
Respiratory infection & 3.19 & $2.07-4.91$ & $<0.001$ & 3.34 & $1.98-5.65$ & $<0.001$ \\
Delta SOFA score $\geq 2$ & 4.19 & $2.65-6.63$ & $<0.001$ & 2.83 & $1.65-4.83$ & $<0.001$ \\
Lactate $\geq 2$ mmol/L & 4.60 & $2.95-7.17$ & $<0.001$ & 3.37 & $2.04-5.57$ & $<0.001$ \\
Procalcitonin $>0.5 \mathrm{ng} / \mathrm{mL}$ & 2.49 & $1.61-3.84$ & $<0.001$ & 0.99 & $0.57-1.71$ & 0.967
\end{tabular}

${ }^{*}$ Malignancy refers to metastatic solid cancer or hematologic malignancy. $p$-value of goodness-of-fit with the Hosmer-Lemeshow test $=0.7595$. OR odds ratio, CI confidence interval, SOFA Sequential Organ Failure Assessment.

\subsection{Discriminating Ability of Presepsin for 28-Day Mortality Based on Delta SOFA Score and} Lactate Level

The 28-day mortality was $5.4 \%(n=23 / 428)$ in the low presepsin group $(\leq 755 \mathrm{pg} / \mathrm{mL})$ and $24.2 \%(n=79 / 327)$ in the high presepsin group $(>755 \mathrm{pg} / \mathrm{mL})(p<0.001)$. The 28-day mortality was significantly different between the low presepsin group and the high presepsin group, even in subgroups including non-sepsis patients with delta SOFA $<2$ ( $3.6 \%$ vs. $13.3 \%, p<0.001)$ and patients with low lactate levels $<2 \mathrm{mmol} / \mathrm{L}(2.7 \%$ vs. $14.4 \%$, $p<0.001$ ) (Figure 3). 

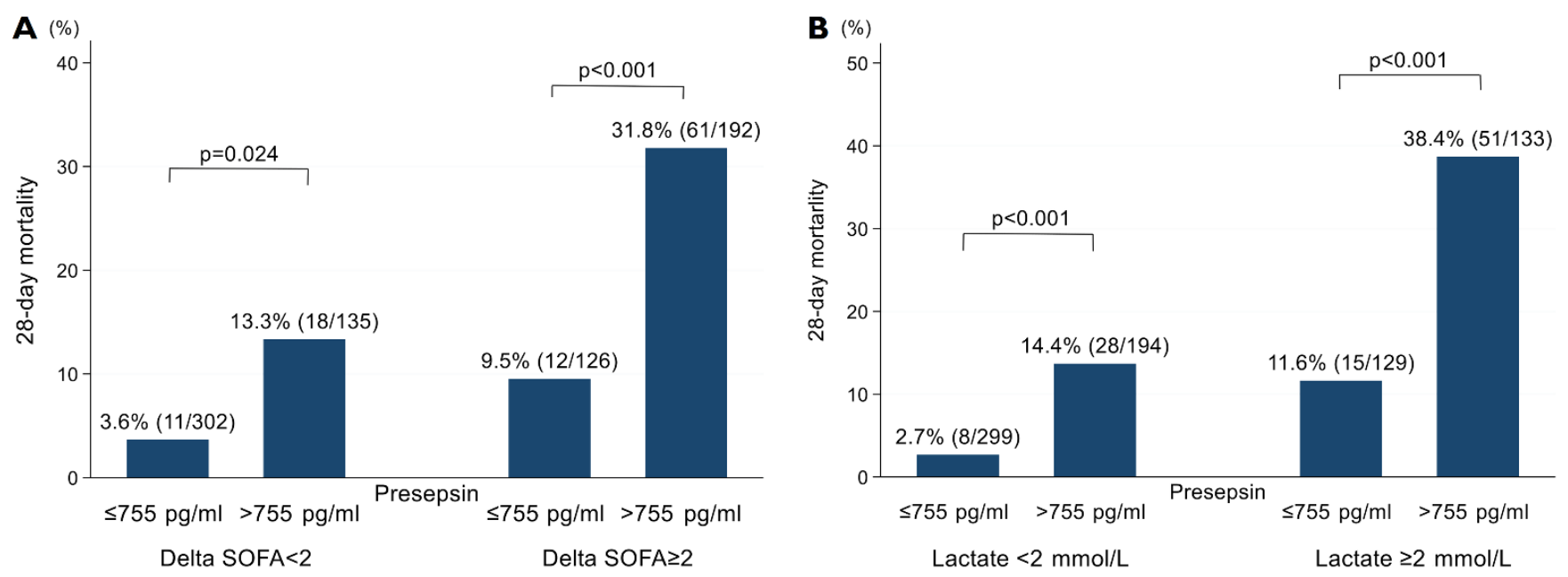

Figure 3. Comparison of 28-day mortality according to the level of presepsin based on (A) delta sofa score and (B) lactate value, respectively. SOFA, Sequential Organ Failure Assessment.

The AUC of presepsin for predicting 28-day mortality was 0.747 (95\% CI: 0.701-0.792). (Figure 4), which was significantly higher than that of PCT (AUC: 0.678, 95\% CI: 0.630-0.725) (Supplementary Figure S1). The AUC was similar between non-sepsis patients with delta SOFA score $<2(0.755,95 \%$ CI: 0.701-0.792) and sepsis patients with delta SOFA $\geq 2$ $(0.709,95 \%$ CI: $0.635-0.783)$. This trend was also consistent when the patients were divided according to the lactate level $(<2 \mathrm{mmol} / \mathrm{L} \mathrm{vs}$. $\geq 2 \mathrm{mmol} / \mathrm{L})$.

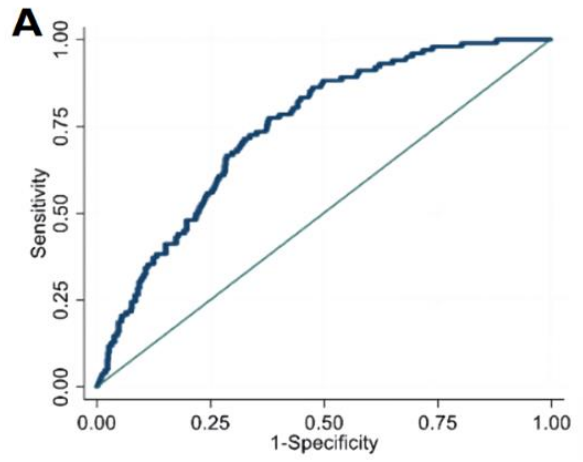

Overall presepsin: AUC, 0.747 (95\% CI: 0.701-0.792)

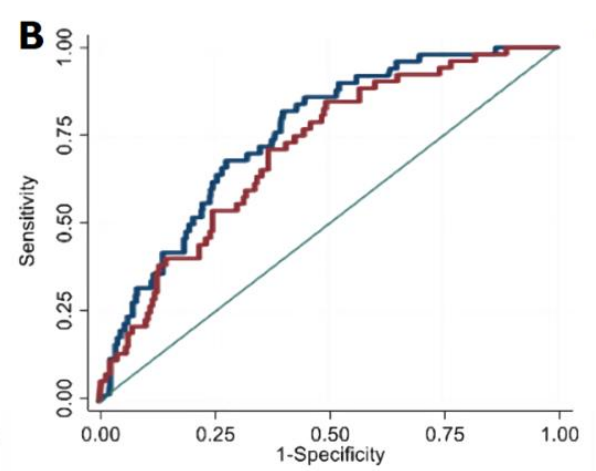

$-\triangle$ SOFA < 2: AUC, $0.755(95 \% \mathrm{Cl}: 0.692-0.817)$ $-\triangle$ SOFA $\geq 2$ : AUC, $0.709(95 \% \mathrm{Cl}: 0.635-0.783)$

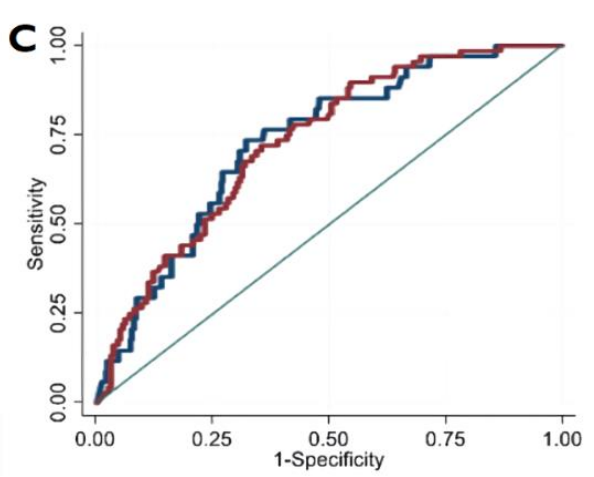

- lactate < 2: AUC, $0.731(95 \% \mathrm{Cl}: 0.652-0.811)$

- lactate $\geq 2:$ AUC, $0.731(95 \% \mathrm{Cl}: 0.667-0.795)$

Figure 4. Area Under the Curve (AUC) of the Receiver Operating Characteristic (ROC) Curves of presepsin for predicting 28-day mortality according to the delta SOFA score and the lactate level in the following groups: (A) overall study population, (B) subgroups according to the delta SOFA ( $<2$ or $\geq 2)$, and (C) subgroups according to the lactate levels $(<2 \mathrm{mmol} / \mathrm{L}$ or $\geq 2 \mathrm{mmol} / \mathrm{L})$.

3.5. Combined Interpretation of the High Presepsin Cut-Off, Delta SOFA Score $\geq 2$ Points, and Lactate $\geq 2 \mathrm{mmol} / \mathrm{L}$

The AUC of presepsin with a cut-off value of $755 \mathrm{pg} / \mathrm{mL}$ in predicting 28-day mortality was 0.697 (95\% CI: $0.653-0.742)$, which was the higher than that of delta SOFA score $\geq 2$ (0.670 [95\% CI: 0.623-0.718]) and lactate $\geq 2$ mmol/L (0.682 [95\% CI: 0.633-0.731]). When presepsin was combined with delta SOFA score and lactate individually, the AUC in predicting 28-day mortality was 0.750 (95\% CI: 0.703-0.797) and 0.772 (95\% CI: 0.727-0.818), respectively, showing a greater AUC than with exclusive use of each criterion (Figure 5). Combining the three criteria exhibited the greatest AUC of 0.795 (95\% CI: 0.749-0.841). 


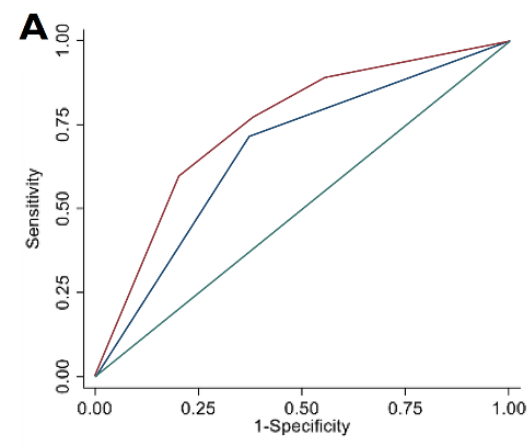

$-\triangle S O F A \geq 2:$ AUC, $0.670(95 \% \mathrm{Cl}: 0.623-0.718)$

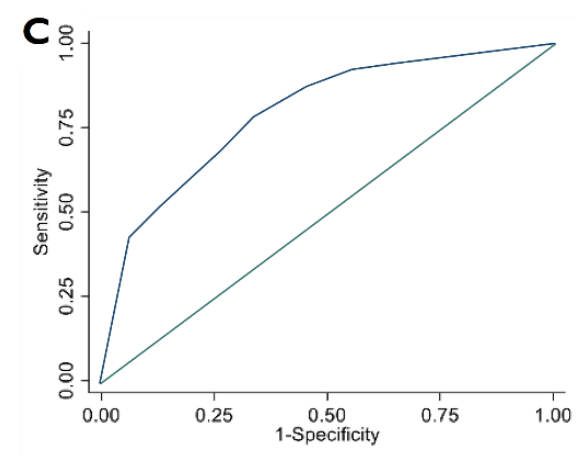

$-\triangle$ SOFA $\geq 2$ and lactate $\geq 2$ and Presepsin $>755$

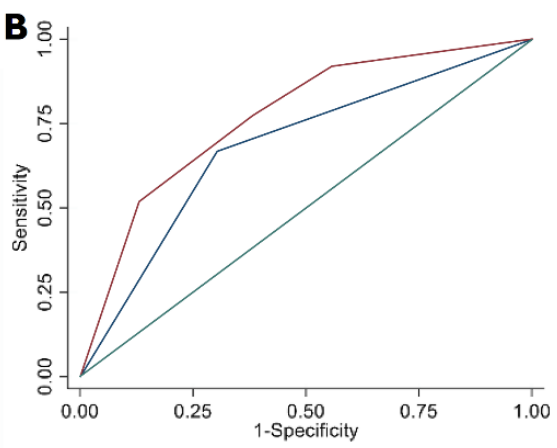

-lactate $\geq 2$ : AUC 0.682 (95\% Cl: 0.633-0.731)

Figure 5. Area under the curve (AUC) of the receiver operating characteristic (ROC) curves of (A) delta SOFA score $\geq 2$ points and combination model with high presepsin, (B) lactate $\geq 2 \mathrm{mmol} / \mathrm{L}$ and combination model with high presepsin, and (C) combination model of delta SOFA score $\geq 2$, lactate $\geq 2 \mathrm{mmol} / \mathrm{L}$, and presepsin. High presepsin was defined as a presepsin level greater than $755 \mathrm{pg} / \mathrm{mL}$.

The sensitivity and specificity of presepsin with a cut-off value of $755 \mathrm{pg} / \mathrm{mL}$ were 77.5\% (95\% CI: 68.1-85.1) and 62\% (95\% CI: 58.2-65.8), respectively (Table 3). If criteria for either presepsin or delta SOFA was fulfilled, greater sensitivity $(89.2 \%, 95 \%$ CI: 81.5-94.5) was obtained compared to using the delta SOFA score alone. Positive criteria for both values increased specificity (79.9\%, 95\% CI: 76.7-82.9). Likewise, adding the lactate criterion or combining all three criteria showed similar trends. The highest sensitivity $(94.1 \%, 95 \% \mathrm{CI}$ : 87.6-97.8) was achieved when the three criteria were combined in an "or" manner; highest specificity (93.7\%, 95\% CI: 91.6-95.5) and PPV (51.8\%, 95\% CI: 40.7-62.7) were achieved with an "and" combination.

Table 3. Prognostic accuracy of presepsin $>755 \mathrm{pg} / \mathrm{mL}$, delta SOFA score $\geq 2$ points, and lactate $\geq 2$ mmol/L for predicting 28-day mortality.

\begin{tabular}{|c|c|c|c|c|}
\hline & $\begin{array}{l}\text { Sensitivity } \\
(95 \% \text { CI) }\end{array}$ & $\begin{array}{l}\text { Specificity } \\
(95 \% \text { CI) }\end{array}$ & $\begin{array}{c}\text { PPV } \\
(95 \% \text { CI })\end{array}$ & $\begin{array}{c}\text { NPV } \\
(95 \% \text { CI })\end{array}$ \\
\hline Presepsin $>755 \mathrm{pg} / \mathrm{mL}$ & $77.5(68.1-85.1)$ & $62(58.2-65.8)$ & $24.2(19.6-29.2)$ & $94.6(92-96.6)$ \\
\hline$\Delta \mathrm{SOFA} \geq 2$ & $71.6(61.8-80.1)$ & $62.5(58.6-66.2)$ & $23(18.4-28)$ & $93.4(90.6-95.5)$ \\
\hline Lactate $\geq 2 \mathrm{mmol} / \mathrm{L}$ & $66.7(56.6-75.7)$ & $69.7(66-73.2)$ & $25.6(20.4-31.2)$ & $93.0(90.4-95.1)$ \\
\hline Presepsin $>755 \mathrm{pg} / \mathrm{mL}$ or $\Delta \mathrm{SOFA} \geq 2$ & $89.2(81.5-94.5)$ & $44.6(40.7-48.5)$ & $20.1(16.5-24.1)$ & $96.4(93.6-98.2)$ \\
\hline Presepsin $>755 \mathrm{pg} / \mathrm{mL}$ or lactate $\geq 2 \mathrm{mmol} / \mathrm{L}$ & $92.2(85.1-96.6)$ & $44.6(40.7-48.5)$ & $20.6(17-24.6)$ & $97.3(94.8-98.8)$ \\
\hline $\begin{array}{l}\text { Presepsin }>755 \mathrm{pg} / \mathrm{mL} \text { or } \Delta S O F A \geq 2 \text { or } \\
\text { lactate } \geq 2 \mathrm{mmol} / \mathrm{L}\end{array}$ & $94.1(87.6-97.8)$ & $34.3(30.7-38.1)$ & $18.3(15.1-21.9)$ & $97.4(94.4-99)$ \\
\hline Presepsin $>755 \mathrm{pg} / \mathrm{mL}$ and $\Delta$ SOFA $\geq 2$ & $59.8(49.6-69.4)$ & $79.9(76.7-82.9)$ & $31.8(25.3-38.9)$ & $92.7(90.3-94.7)$ \\
\hline Presepsin $>755 \mathrm{pg} / \mathrm{mL}$ and lactate $\geq 2 \mathrm{mmol} / \mathrm{L}$ & $52.0(41.8-62.0)$ & $87.1(84.3-89.6)$ & $38.7(30.5-47.4)$ & $92.1(89.7-94.1)$ \\
\hline $\begin{array}{l}\text { Presepsin }>755 \mathrm{pg} / \mathrm{mL} \text { and } \Delta S O F A \geq 2 \text { and } \\
\text { lactate } \geq 2 \mathrm{mmol} / \mathrm{L}\end{array}$ & $43.1(33.4-53.2)$ & 93.7 (91.6-95.5) & $51.8(40.7-62.7)$ & $91.3(89.0-93.4)$ \\
\hline
\end{tabular}

$\Delta$, delta; SOFA, Sequential Organ Failure Assessment; CI, confidence interval; PPV, positive predictive value; NPV, negative predictive value.

\section{Discussion}

Since presepsin was first discovered as a novel biomarker for sepsis in 2002 [13], a number of studies have demonstrated the significance of presepsin for identifying sepsis or predicting mortality. Although the diagnostic and prognostic values of presepsin for sepsis have been demonstrated in various studies [14-17], the complementary utility and applicability of presepsin in clinical practice still need further research.

Sepsis, a life-threatening organ dysfunction caused by infection, continues to be a major cause of death globally [18]. Multiple studies have established the advantage of prompt intervention, such as rapid antibiotics administration, in reducing mortality $[19,20]$. Early recognition of high-risk patients is crucial to improving patient outcomes. Nonetheless, it is often challenging to sort out high-risk patients in real-life clinical practice. 
In this study, our data showed a significant difference in presepsin levels among the non-sepsis, sepsis, and septic shock patients, when categorized by the Sepsis-3 criteria. Regarding previous studies, the results of this study are in line with previous results on the prognostic value of presepsin. While the study was performed in a tertiary hospital with a cancer center, the study population comprised patients with malignancies, those who were immunocompromised, and those with multiple comorbidities. In addition, presepsin levels were significantly different between survivors and non-survivors in both the sepsis and non-sepsis groups. Thus, utilization of presepsin could be helpful in predicting the outcome in both sepsis and non-sepsis patients. Moreover, combining presepsin with other well-known markers, such as the Sepsis-3 definition by SOFA score and lactate, could provide greater predictive power. When presepsin was combined with delta SOFA score, the predictive power for mortality was greater than using SOFA score alone, suggesting the advantage of ancillary use of presepsin along with other markers. Thus, use of presepsin could improve the identification of high-risk patients.

Although the biological function of presepsin itself has yet to be elucidated, the primary source of presepsin secretion is CD14-positive monocyte/macrophage lineages which are triggered by bacterial phagocytosis [21]. Presepsin is currently known to rise faster than PCT and CRP $[6,7,10]$. It is also speculated that presepsin rises faster than lactate, considering the fact that increases in lactate level result from tissue hypoperfusion. Besides, presepsin shows excellent correlation between sample types including whole blood, and the result can be obtained within $17 \mathrm{~min}$ [11]. Hence, presepsin is a feasible marker for predicting mortality in sepsis, especially at an early stage before progression of organ failure. Utilization of presepsin may assist in identifying patients with potentially unfavorable outcomes and could improve mortality rates by facilitating prompt treatment.

Although other markers such as CRP and PCT have been widely used, those markers have their own limitations in terms of predicting prognosis [22]. Lactate levels could be also increased by hepatic dysfunction, renal dysfunction, or catecholamine, as well as sepsis-induced tissue hypoxia [23]. Compared with those biomarkers, presepsin might have some advantages in that it could be more specific for sepsis and could have an earlier increase. On the other hand, presepsin may be elevated in renal or hepatic dysfunction without infection $[3,24]$ so further research would be needed for clinical interpretation.

There are several limitations to our study that deserve to be acknowledged. First, while the subjects were enrolled in a prospective manner, the selection criteria included procalcitonin and blood culture, which could cause selection bias. However, since procalcitonin and blood culture are generally ordered for almost all suspected sepsis patients, the chance of selection bias is relatively low. Besides, to the best of our knowledge, the number of reviewed subjects in this study is the largest among studies evaluating presepsin under the Sepsis-3 criteria, to date. Second, this study was conducted in a single institution, and approximately two-thirds of the enrolled subjects had a malignancy. Since the population of our study tended to be biased toward high severity, further research should be carried out to ascertain the prognostic value of presepsin in patients with less severe illness. Third, while various outcome measures for sepsis research have been proposed [25], only a single outcome measure, 28-day mortality, has been evaluated. Although 28-day mortality has been widely used as the primary measure representing the clinical outcome of sepsis, other patient-centered outcomes including long-term survival could potentially differ from 28-day mortality and further studies should investigate various sepsis outcomes to evaluate the clinical utility of presepsin [25]. Fourth, we were unable to obtain follow-up presepsin level changes over time. Since we only retrieved the presepsin level from a single time point at presentation, the actual onset of sepsis and whether the measured presepsin level represents the peak level are uncertain. Nonetheless, the characteristics of our data may better reflect the real-world clinical setting. Further research regarding the underlying mechanism of presepsin secretion, the biological role of presepsin, and the serial kinetics of presepsin according to the patient status are warranted. 


\section{Conclusions}

Presepsin measurement had prognostic value for predicting 28-day mortality both in non-sepsis patients and sepsis patients. Complementary use of presepsin with the SOFA score of the Sepsis-3 clinical criteria or with the lactate level may identify more high-risk septic patients and provide useful prognostic information.

Supplementary Materials: The following are available online at https://www.mdpi.com/article/ 10.3390/biomedicines9091076/s1, Supplementary Table S1: Clinical characteristics of the patients with non-sepsis, sepsis, and septic shock, Supplementary Figure S1: Comparisons of the receiver operating characteristic (ROC) curves of presepsin and procalcitonin for predicting 28-day mortality: (a) overall group, (b) non-sepsis patients, and (c) sepsis patients.

Author Contributions: Study concepts and design, E.-S.K., T.G.S., C.-M.P. and C.W.J.; literature search, J.E.P., B.L., M.-J.A. and S.J.Y.; database development, E.-S.K., J.E.P., T.G.S. and S.J.Y.; data collection, all authors; technical support, E.-S.K. and H.-D.P.; statistical analysis, J.E.P. and T.G.S.; manuscript preparation, J.E.P., B.L., T.G.S. and E.-S.K.; figures, J.E.P. and S.Y.H.; final manuscript review, all authors. All authors have read and agreed to the published version of the manuscript.

Funding: This research received no external funding.

Institutional Review Board Statement: The study was conducted according to the guidelines of the Declaration of Helsinki, and approved by the Institutional Review Board of Samsung Medical Center (IRB No. SMC-2019-05-170).

Informed Consent Statement: The Institutional Review Board of Samsung Medical Center waived the need for informed consent since this study was anonymous and utilized residual samples.

Data Availability Statement: The datasets used and/or analyzed during the current study are available from the corresponding author upon reasonable request.

Acknowledgments: We would like to thank H.J. Shon and M.J. Jung for their technical support.

Conflicts of Interest: The authors declare no conflict of interest.

\section{Abbreviations}

ROC Receiver operating characteristic

AUC Area under the curve

IQR Interquartile range

SOFA Sequential organ failure assessment

PCT Procalcitonin

CRP C-reactive protein

LPS Lipopolysaccharide

ED Emergency department

ICU Intensive care unit

PST Plasma separating tube

ALP Alkaline phosphatase

QC Quality control

$\mathrm{CV} \quad$ Coefficient of variation

CI Confidence interval

MAP Mean arterial pressure

PPV Positive predictive value

NPV Negative predictive value

COVID-19 Coronavirus disease 2019

OR Odds ratio

aOR Adjusted odds ratio 


\section{References}

1. Vincent, J.L.; Jones, G.; David, S.; Olariu, E.; Cadwell, K.K. Frequency and mortality of septic shock in Europe and North America: A systematic review and meta-analysis. Crit. Care 2019, 23, 196. [CrossRef]

2. Singer, M.; Deutschman, C.S.; Seymour, C.W.; Shankar-Hari, M.; Annane, D.; Bauer, M.; Bellomo, R.; Bernard, G.R.; Chiche, J.D.; Coopersmith, C.M.; et al. The Third International Consensus Definitions for Sepsis and Septic Shock (Sepsis-3). JAMA 2016, 315, 801-810. [CrossRef] [PubMed]

3. Zhu, Y.; Li, X.; Guo, P.; Chen, Y.; Li, J.; Tao, T. The accuracy assessment of presepsin (sCD14-ST) for mortality prediction in adult patients with sepsis and a head-to-head comparison to PCT: A meta-analysis. Ther. Clin. Risk Manag. 2019, 15, 741-753. [CrossRef]

4. Heilmann, E.; Gregoriano, C.; Schuetz, P. Biomarkers of Infection: Are They Useful in the ICU? Semin. Respir. Crit. Care Med. 2019, 40, 465-475. [CrossRef] [PubMed]

5. Yang, H.S.; Hur, M.; Yi, A.; Kim, H.; Lee, S.; Kim, S.N. Prognostic value of presepsin in adult patients with sepsis: Systematic review and meta-analysis. PLoS ONE 2018, 13, e0191486. [CrossRef] [PubMed]

6. Yaegashi, Y.; Shirakawa, K.; Sato, N.; Suzuki, Y.; Kojika, M.; Imai, S.; Takahashi, G.; Miyata, M.; Furusako, S.; Endo, S. Evaluation of a newly identified soluble CD14 subtype as a marker for sepsis. J. Infect. Chemother. 2005, 11, 234-238. [CrossRef]

7. Shozushima, T.; Takahashi, G.; Matsumoto, N.; Kojika, M.; Okamura, Y.; Endo, S. Usefulness of presepsin (sCD14-ST) measurements as a marker for the diagnosis and severity of sepsis that satisfied diagnostic criteria of systemic inflammatory response syndrome. J. Infect. Chemother. 2011, 17, 764-769. [CrossRef]

8. Kahveci, U.; Ozkan, S.; Melekoglu, A.; Usul, E.; Ozturk, G.; Cetin, E.; Abatay, K.; Sahin, A. The role of plasma presepsin levels in determining the incidence of septic shock and mortality in patients with sepsis. J. Infect. Dev. Ctries 2021, 15, 123-130. [CrossRef] [PubMed]

9. Masson, S.; Caironi, P.; Spanuth, E.; Thomae, R.; Panigada, M.; Sangiorgi, G.; Fumagalli, R.; Mauri, T.; Isgro, S.; Fanizza, C.; et al. Presepsin (soluble CD14 subtype) and procalcitonin levels for mortality prediction in sepsis: Data from the Albumin Italian Outcome Sepsis trial. Crit. Care 2014, 18, R6. [CrossRef]

10. Koizumi, Y.; Shimizu, K.; Shigeta, M.; Okuno, T.; Minamiguchi, H.; Kito, K.; Hodohara, K.; Yamagishi, Y.; Andoh, A.; Fujiyama, Y.; et al. Plasma presepsin level is an early diagnostic marker of severe febrile neutropenia in hematologic malignancy patients. BMC Infect. Dis. 2017, 17, 27. [CrossRef] [PubMed]

11. Okamura, Y.; Yokoi, H. Development of a point-of-care assay system for measurement of presepsin (sCD14-ST). Clin. Chim. Acta 2011, 412, 2157-2161. [CrossRef]

12. DeLong, E.R.; DeLong, D.M.; Clarke-Pearson, D.L. Comparing the areas under two or more correlated receiver operating characteristic curves: A nonparametric approach. Biometrics 1988, 44, 837-845. [CrossRef] [PubMed]

13. Okamura, Y. Usefulness of Presepsin Measurement: A New Biomarker for Sepsis. Rinsho Byori Jpn. J. Clin. Pathol. 2015, 63, 62-71.

14. Wu, J.; Hu, L.; Zhang, G.; Wu, F.; He, T. Accuracy of Presepsin in Sepsis Diagnosis: A Systematic Review and Meta-Analysis. PLoS ONE 2015, 10, e0133057. [CrossRef] [PubMed]

15. Zhang, J.; Hu, Z.D.; Song, J.; Shao, J. Diagnostic Value of Presepsin for Sepsis: A Systematic Review and Meta-Analysis. Medicine 2015, 94, e2158. [CrossRef]

16. Zhang, X.; Liu, D.; Liu, Y.N.; Wang, R.; Xie, L.X. The accuracy of presepsin (sCD14-ST) for the diagnosis of sepsis in adults: A meta-analysis. Crit. Care 2015, 19, 323. [CrossRef] [PubMed]

17. Zheng, Z.; Jiang, L.; Ye, L.; Gao, Y.; Tang, L.; Zhang, M. The accuracy of presepsin for the diagnosis of sepsis from SIRS: A systematic review and meta-analysis. Ann. Intensive Care 2015, 5, 48. [CrossRef] [PubMed]

18. Rudd, K.E.; Johnson, S.C.; Agesa, K.M.; Shackelford, K.A.; Tsoi, D.; Kievlan, D.R.; Colombara, D.V.; Ikuta, K.S.; Kissoon, N.; Finfer, S.; et al. Global, regional, and national sepsis incidence and mortality, 1990-2017: Analysis for the Global Burden of Disease Study. Lancet 2020, 395, 200-211. [CrossRef]

19. Seymour, C.W.; Gesten, F.; Prescott, H.C.; Friedrich, M.E.; Iwashyna, T.J.; Phillips, G.S.; Lemeshow, S.; Osborn, T.; Terry, K.M.; Levy, M.M. Time to Treatment and Mortality during Mandated Emergency Care for Sepsis. N. Engl. J. Med. 2017, 376, 2235-2244. [CrossRef]

20. Liu, V.X.; Fielding-Singh, V.; Greene, J.D.; Baker, J.M.; Iwashyna, T.J.; Bhattacharya, J.; Escobar, G.J. The Timing of Early Antibiotics and Hospital Mortality in Sepsis. Am. J. Respir. Crit. Care Med. 2017, 196, 856-863. [CrossRef] [PubMed]

21. Arai, Y.; Mizugishi, K.; Nonomura, K.; Naitoh, K.; Takaori-Kondo, A.; Yamashita, K. Phagocytosis by human monocytes is required for the secretion of presepsin. J. Infect. Chemother. 2015, 21, 564-569. [CrossRef] [PubMed]

22. Steinbach, G.; Bolke, E.; Grunert, A.; Storck, M.; Orth, K. Procalcitonin in patients with acute and chronic renal insufficiency. Wien. Klin. Wochenschr. 2004, 116, 849-853. [CrossRef]

23. Jeppesen, J.B.; Mortensen, C.; Bendtsen, F.; Moller, S. Lactate metabolism in chronic liver disease. Scand. J. Clin. Lab. Investig. 2013, 73, 293-299. [CrossRef] [PubMed]

24. Nakamura, Y.; Ishikura, H.; Nishida, T.; Kawano, Y.; Yuge, R.; Ichiki, R.; Murai, A. Usefulness of presepsin in the diagnosis of sepsis in patients with or without acute kidney injury. BMC Anesth. 2014, 14, 88. [CrossRef]

25. Marshall, J.C.; Vincent, J.L.; Guyatt, G.; Angus, D.C.; Abraham, E.; Bernard, G.; Bombardier, C.; Calandra, T.; Jorgensen, H.S.; Sylvester, R.; et al. Outcome measures for clinical research in sepsis: A report of the 2nd Cambridge Colloquium of the International Sepsis Forum. Crit. Care Med. 2005, 33, 1708-1716. [CrossRef] [PubMed] 